\title{
Diagnóstico para implementar servicios automotrices y línea blanca en la parroquia Barbones cantón El Guabo
}

\section{Diagnostic to implement automotive services and appliances in the neighborhood Barbones in the area El Guabo}

Cuenca García Paola Vanessa, Hurtado Jiménez Silvana Yajaira, Cartuche Paqui David Vladimir. Universidad Técnica de Machala, Ecuador Autor para correspondencia: syhurtado_est@utmachala.edu.ec Fecha de recepción: 25 de Octubre de 2017 - Fecha de aceptación: 15 de Mayo de 2018

Resumen: Esta investigación tiene como objetivo conocer cuáles son los servicios automotrices y de línea blanca que demanda la Parroquia Barbones del Cantón El Guabo, los resultados permiten evaluar la oferta existente en el sector para determinar cuáles de estos servicios son los que deben mejorar e incrementar, contribuyendo a la vez con dar paso a nuevas plazas de trabajo y mejorar la actividad económica de los habitantes de esta parroquia. Esta investigación es de carácter exploratoria ya que busca información para lograr encontrar la solución, la muestra para la encuesta está conformada por 1553 hogares de la Parroquia Barbones, el resultado del tamaño de la muestra con un error de 0.05 da un total de 318 hogares, los datos de la población se obtienen a través del Instituto Nacional de Estadística y Censos (INEC), también se utiliza la observación y la entrevistas a los 5 propietarios de talleres artesanales que realizan actividades similares a lo antes mencionado. La técnica de recolectar información es a través de un cuestionario estructurado con preguntas abiertas para la entrevista, y, con preguntas cerradas y de opción múltiple para la encuesta. A través de estos cuestionarios se conoció que el 100\% de la población demanda en servicios para $\mathrm{ABC}$ motor de inyección, y electromecánica siendo esta la mejor opción para implementar y mejorar en los talleres artesanos existentes, y, en arreglos de línea blanca el 80\% requiere la reparación de refrigeradoras, de cocinas eléctricas y a gas.

Palabras Claves: implementar; tecnología; taller; servicios; empresa

Abstract: The purpose of this investigation is to know what automotive and white line services are needed in the parish Barbones of Canton El Guabo, and with the results of the investigation is allowed to evaluate the existing offer in this sector, whit purpose to confirm which services should be improved and increased, in the same time also can provide new jobs and improve the economy activity of the residents in the parish. The character of this investigation is exploratory, because collect data is to find the solution and 1553 homes of the Barbones parish are the samples of the investigation, the result of the sample size with an error of 0.05 gives a total of 318 households, by the national Institute of Static and Census got the data of the population, also it's used the observation and interview for 5 owners of craft workshops performed the similar activities in the above. Through a structured questionnaire with open and closed questions for the interview and 
multiple choices for the survey, is the technique of the collecting information. Through these questionnaires is known that $100 \%$ of the population needs $\mathrm{ABC}$ motors injection services, and the best option to implement and improve in the existing craft workshops is electromechanical, and $80 \%$ of the white line to be repaired are refrigerators, electric and gas cookers.

Key Words: implement; technology; workshop; services; company

\section{Introducción}

Una empresa siempre está en constante cambio, es decir siempre buscar mejoraras para su negocio, si ofrece algún tipo de producto o servicio la empresa analiza el entorno que los rodea con el fin de ver que nuevos productos ofrecer y que cambios implementar, para todo esto hoy en día se utiliza la tecnología para cumplir con las expectativas de los consumidores y proporcionar competitividad a la misma.

Acs \& kallas, (2007) establece que mediante evidencia empírica ha demostrado que las Mipymes (micro, pequeñas y medianas empresas) son las que activan la economía del país. Las Mipymes en Latinoamérica juegan un papel muy importante en la sociedad, son quienes proporcionan empleo y se encuentran en un promedio de $99,12 \%$ del total de empresas en Latinoamérica, para el caso de estudio se tomó en cuenta a países como: Argentina, Bolivia, Brasil, Chile, Colombia, Costa Rica, El Salvador Guatemala, México, Panamá, Perú, Uruguay y Venezuela, se establece que las empresas ubicadas en estos países alcanzan un promedio de $64,26 \%$ del total de empleos proporcionados, y distribuyéndolas sectorialmente se concluye que el sector comercial predomina con un promedio de $49 \%$, el sector de servicios alcanza $38 \%$,y, el sector manufacturero con un $13 \%$. (Saavedra G \& Hernandéz C, 2008)

Según Pastor, Escobar, Mayoral, \& Ruiz (2010) sotiene que la tecnología es aquella que utiliza y aprovecha todos los conocientos científicos y técnicos para que el ser humano lo ponga en práctica en cualquier tiempo (p. 295).

\section{"La tecnología refleja en qué trabajan las personas y que elementos lo utilizan} para realizar ese trabajo" (Krajewski \& Ritzman, 2000, p.127).

En el mundo la tecnología avanza a pasos agigantados y al ser humano le presentan ideas que lo ayuden a mejorar sus negocios, buscando siempre agilizar sus procesos. Hablamos de la tecnología en los negocios pero, ¿Qué negocios se pueden utilizar la tecnología?, en si la tecnología está relacionada con todo tipo de negocio sin excepción alguna.

"La tecnología como como conocimiento práctico, Objetos físicos y los procedimientos que se usan para generar productos o servicios" (Krajewski \& Ritzman, 2000, p. 127).

Para esta investigación analizamos cuales son los servicios automotrices y de línea blanca que se debe implementar en la parroquia Barbones del cantón El Guabo, en la cual a su vez relacionamos con el tema de la tecnología debido a que estas empresas que ofrecen servicios mecánicos la utilizan y es de gran ayuda para agilizar los procesos en cada actividad realizada. 
Técnicamente un taller engloba diferentes significados, como un simposio se convierte en un taller cuando los asistentes ejecutan prácticas del tema que se ha tratado, también es entendido como un espacio físico en el que se ejecutan operaciones manualmente o artesanalmente, por ejemplo un taller de zapatos, otro de los significados se relaciona con un taller de soldadura, de mecánica la cual se direcciona por la reparación de máquinas averiadas, como vehículos, electrodomésticos, etc. (Antiche, 2012)

"Se entiende por reparación de vehículos automóviles y de sus equipos y componentes, aquellos establecimientos industriales en los que se efectúen operaciones encaminadas a la restitución de las condiciones normales del estado y funcionamiento del vehículo automóvil”. (Bernardo Hernando, 2008)

Taller mecánico está direccionado a la reparación de automóviles, está conformador por maquinaria tecnológica y herramientas que sirven para optimizar el trabajo realizado por el encargado de reparar el daño, este a la vez debe tener conocimientos acerca de cómo operar estas máquinas y herramientas. Las máquinas de un taller mecánicas están destinadas directa o indirectamente a la manufactura. (Chacón A., 2004)

La industria automotriz crece conforme la tecnología evoluciona y conforme crecen los mercados emergentes, con su crecimiento da paso a recepción de mano de obra calificada para realizar actividades específicas en la reparación de automóviles. Debido al crecimiento de la industria automotriz el Ecuador ha tenido un gran desarrollo con estas tecnologías y por ende es unos de los factores que contribuyen al crecimiento de la economía del país, las ensambladoras del país debido sus maquinarias y herramientas, son reconocidas por sus productos de alta calidad en el mercado nacional e internacional (Ecuador, 2017)

"El sector automotor tiene una participación importante en la economía del país debido a los ingresos que genera en todas las actividades económicas directas e indirectas que involucran" (Ecuador, Análisis del sector automotriz, 2013)

En los resultados de censo de INEC se evidenció que en el año 2014 existían 29.068 empresas que están enfocadas en actividades con el sector automotor, es decir el: " $70 \%$ de empresas están direccionados al manteniendo y reparación de vehículos, y el 30\% restante del sector automotor está enfocado en la venta de partes, piezas y accesorios de vehículos y venta al por menos de combustible y vehículos” (Quisiguiña, 2016)

Según española, s.n menciona que los servicios son aquellas ocupaciones, pestación o asisencia que ofrece una organización a sus clientes en un tiempo determinado, los consumidores son quienes califican la calidad de servicio que han recibido. Quiñones \& Aldana de Vega, (2006) En su libro Calidad y Servicio, menciona un concepto de F. Lamata, donde conceptualiza al servicio como un proceso producido por el hombre (producto) que soporta un valor de utilidad ( resuelve un problema o satisface una necesidad) que puede y suele cambiarse por otros bienes o servicios o por su valor en moneda (p.58). 
(...) fue concebido como una utilidad en sí misma que en oportunidades; requiere presentarse a través de bienes tangibles y, en otras, por medio de la comunicación cliente-proveedor (Aldana de Vega, 2011, p. 75)

Cuando hablamos de servicios hacemos referencia a la satisfacción que recibe el cliente de un servicio adquirido este no puede ser divisado como un producto, pero se puede medir mediante la satisfacción del cliente es ahí cuando la empresa determina cuan agradable fue el servicio prestado y que se medidas tomar para mejorar dicho servicio.

Para el estudio del tema mencionado identificamos dos hipótesis la primera a considerar es que la parroquia Barbones del cantón El Guabo está en condiciones para ofrecer servicios automotrices y de línea blanca, la segunda hipótesis a relacionar con el proyecto es la capacitación técnica de la comunidad local es mínima respecto al tema relacionado con las reparaciones de electrodomésticos y automóviles.

Para la problemática se toma en consideración a la parroquia de Babones con apenas 5.137 habitantes de los cuales 1.553 son familias, dato encontrado en GADPT, 2015. Barbones se encuentra rodeada al Oeste por el océano pacifico, limita al norte con la parroquia Tendales, al sur con la ciudad de Machala y al Este con la Cabecera Cantonal de El Guabo (BARBONES, s.f.). Esta parroquia es muy concurrida por diferentes tipos de vehículos de transporte debido a que se encuentra rodeada por haciendas bananeras, la fábrica Industrial Borja y también se encuentra la playa Bajo Alto muy conocida y visitada por varios turistas, es por ello y por los diferentes requerimientos de servicios que han sido solicitados en los últimos años y por ende se realiza el sondeo para conocer qué servicios con favorables brindar en la parroquia.

Uno de los objetivos primordiales que se debe conocer en todo proyecto de emprendimiento y que como autores propios damos énfasis y de la cual se ha direccionado este documento es indagar en la necesidad de servicios que la población requiere a base de la capacidad y conocimiento de quienes requieran esta información, esto causaría que como emprendedores poder ofertar nuestros conocimientos a dicha población.

\section{Materiales y métodos}

Las metodologías para una investigación varían ya que dependen de la información que se desea investigar para que los datos obtenidos puedan ser una base confiable. Según Guerrero (2016), infatiza que la metodología es aquella que nos representa la manera de organizar el proceso de la investigación, de controlar sus resultados y de presentar posibles soluciones a un problema.

"La metodología se utiliza frecuente en estudios doctorales de filosofía, epistemología, educación y lingüística, entre otras disciplinas se caracterizan por su riqueza en descripción y análisis en enfoques cualitativo y cuantitativo, para el desarrollo de una investigación” (HernandezSampieri, 2014).

La investigación tiene como objeto recolectar la información verás para detectar que servicios automotrices y de línea blanca, tienen la mayor demanda en la parroquia. De acuerdo a 
los criterios de Malhotra (2016), tomando en cuenta que el tipo de investigación es la exploratoria, ya, que tiene como objetivo principal la búsqueda de información para la solución a un problema, además se tomará el tipo de muestreo por área a 318 familia, dicho resultado es de acuerdo a la fórmula del tamaño muestral.

$$
\begin{aligned}
& \text { Ecuación 1: Formula muestral para el Universo } \\
& \qquad \mathbf{T M}=\frac{\mathrm{N}}{1+(\mathrm{E})^{2} \mathrm{~N}}=\frac{\mathbf{1 5 5 3}}{1+3.8825}=\mathbf{3 1 8}
\end{aligned}
$$

- $\mathrm{TM}=$ Tamaño de la muestra

- $\mathrm{N}=$ Población

- $\mathrm{E}=$ Grado de error $(0,05)$.

- $1=$ constantes

La técnica de recopilación de información es la encuesta, la entrevista y Observación:

Según, Malhotra (2016) menciona que las técnicas de encuesta permitira obtener información especifica de los participantes, es decir que las encuestas son aquellas que nos permiten obtener información actualizada de lo que se desea conocer, mediante diferente preguntas dando como resultado de forma cuantitativa.

La encuesta se ha dividido por las siguiente zonas o cuidadelas que pertenecen a la parroquia como son:

Tabla 1. Tabla de zonas encuestadas en la parroquia Barbones

\begin{tabular}{lcc}
\hline \multicolumn{1}{c}{ ZONA } & NUMEROS DE ENCUESTAS & EDAD PROMEDIO \\
\hline Calle seis de agosto & 80 & 22 a 40 \\
Manuel Perez y Santa Rosa, & 96 & 18 a 35 \\
Samuel Quimi & 96 & 25 a 50 \\
Brisas Barboneñas & 46 & 20 a 37 \\
\multicolumn{1}{c}{ TOTAL } & $\mathbf{3 1 8}$ & \\
\hline
\end{tabular}

Fuente: Autores

Las familias encuestadas están en una edad promedio de 18 a 50 años, para realizar la recolección de información se tomó en cuenta cuántas veces y cuáles son los servicios que mayormente demandan, que talleres prefieren para realizar la reparación de automotores y línea blanca. Lo que denota la encuesta es que los ciudadanos residentes de la parroquia tienen mayor confianza en el Taller Barbones debido a su transcendencia en el tiempo, pero les gustaría que el mismo amplíe el local, inserte nuevos servicios o que los dueños de los diferentes talleres se unan, para brindar en un solo lugar los servicios requeridos. Según López, López, \& Bernabeu, (2012), la entrevista no es más que una conversación entre personas cuyo fin es conocer las a fondo al individuo entrevistado ya sea para uso académico o personal

"La entrevista es un proceso de interacción humana dentro del cual hay que esforzarse en ser lo más sincero y genuino que se pueda” (Avecevo Ibañez \& López M., 2000, p. 103). 
La observavion nos permite analizar el espacio fisico a investigar visualmente y bajo la prespectiva de quien lo hace para tomar decisiones que favorescan o no a una investigación.

Gallardo Vázquez \& Camacho Herrera, (2008), menciona que la observacion es un estudio pasajero de todo lo que esta pasando en el exterior y no puede interferir en ello ni probocar algun suceso (p.56).

"La observacion se caracteriza por la focalización de la atención de quien la realiza en una parte de la realiddad que estudia, de manera intencional" (Borda Pérez, Tuesca Molina, \& Navarro Lechuga, 2013, p.15).

La entrevista se realizó a los propietarios de los talleres que ofrecen servicios relacionados con automóviles y línea blanca, las actividades que mayormente ejecutan estos pequeños empresarios son arreglos de llantas para vehículos, arreglo de cocina gas, latería de vehículos y soldadura. Los datos fueron obtenidos por la observación y entrevista, ya que se recorrió el sector y se determinó que existen 5 empresas que brindan diferentes tipos de servicios, tres de ellas se encuentran con los permisos legales para realizar su actividad, el restante no tiene estos requisitos para operar.

Tabla 2

Tabla de nombres de las empresas y sus actividades económicas

\begin{tabular}{lc}
\hline Nombre de la Empresa & Actividad \\
\hline Vulcanizadora & Arreglos de llantas y tubos para carros y motonetas, arreglos de cocina a gas, \\
Barbones & soldadura. \\
El vecino & Trabajos en soladuras, y elaboracion de puertas y ventanas de latón y de acero. \\
Taller Aquino & Taller de lateria de vehiculos. \\
Taller Paucar & Arreglos de llantas de motos y su electricidad. \\
Taller Toyita & Arreglos de refigeración en neveras y aires acondicionados. \\
\hline
\end{tabular}

Fuente: Autores

\section{Resultados y discusión}

A través de las encuestas realizadas a la parroquia Barbones se identificó los servicios que mayor demanda tienen, es por ello que este método nos brinda la información necesaria para resolver las hipótesis planteadas al inicio del documentos siendo la primera: la parroquia Barbones del cantón El Guabo está en condiciones para ofrecer servicios automotrices y de línea blanca, esta hipótesis es verdadera debido que la parroquia es muy concurrida y visitada por diferentes turistas y porque se encuentra cerca de empresas bananeras, además según los datos ofrecidos y tabulados mencionan que la población demanda con celeridad los servicios como, arreglos de vehículos en $\mathrm{ABC}$ motor de inyección, servicios electromotrices, suspensión, Balanceo y alineación de neumáticos, frenos, cambio e aceite y filtrado, dirección, transmisión, fresado, también se identificó que es necesario implementar arreglos de líneas blancas y como dato adicional la existencia de demanda en el servicio de soldaduras. 


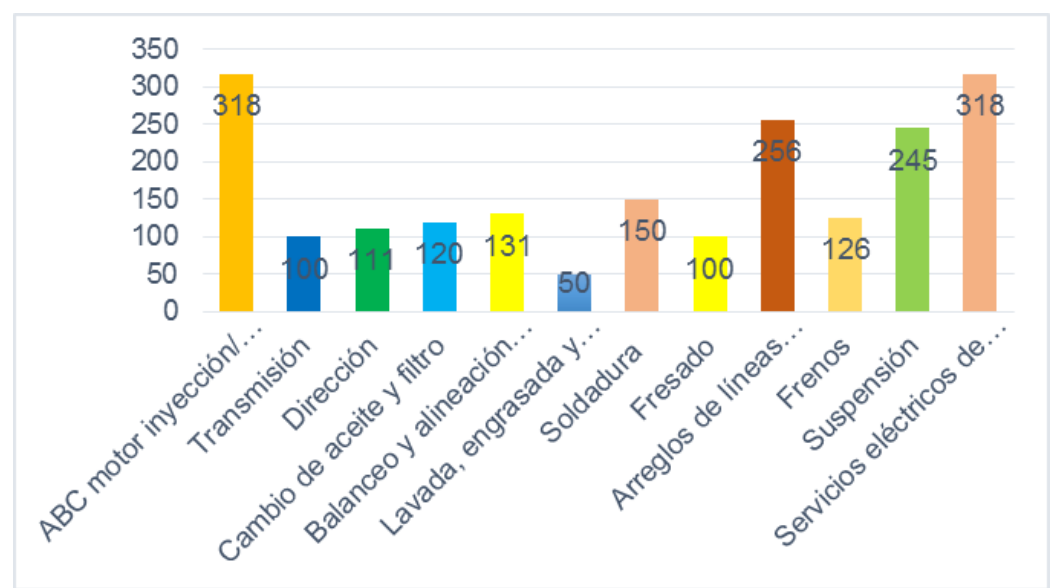

Gráfico 1. Servicios que la población desea Fuente: Autores

La segunda hipótesis a relacionar con el proyecto es que la capacitación técnica de la comunidad local es mínima respecto al tema relacionado con las reparaciones de electrodomésticos y automóviles, esta hipótesis es verdadera, debido a que la población no cuenta con conocimientos técnicos en este tema, en la actualidad Barbones tiene maestros con esta actividad económica pero la gran parte de ellos han estudiado empíricamente y la otra parte ha formado parte de algún centro de capacitación o instituto, este dato es obtenido mediante la entrevista realizada a los cinco propietarios y sus trabajadores de los negocios existentes. Además en la entrevista se analizó que las empresas que existen en la parroquia Barbones cuentan con espacios muy reducidos para brindar esto servicios y comodidad a los clientes, ocasionando muchas veces que estos sean atendidos ocupando la calle, produciendo incomodidad a vehículos y personas que transitan estos lugares.

Para buscar más informacion en la encuenta se realizó la siguiente pregunta de que ¿Qué aspectos considera importantes para visitar un taller ya sea de mecánica, automotriz, entre otros? Calificación en un rango del 1 (menor) al 5 (mayor) importancia, 318 hogares encuenadas consideran que las principales caraceristicas importantes es la calidad del servicio, garantia de la mano de obra, la cercanía, equipos adecuados y la facilidad de pago.

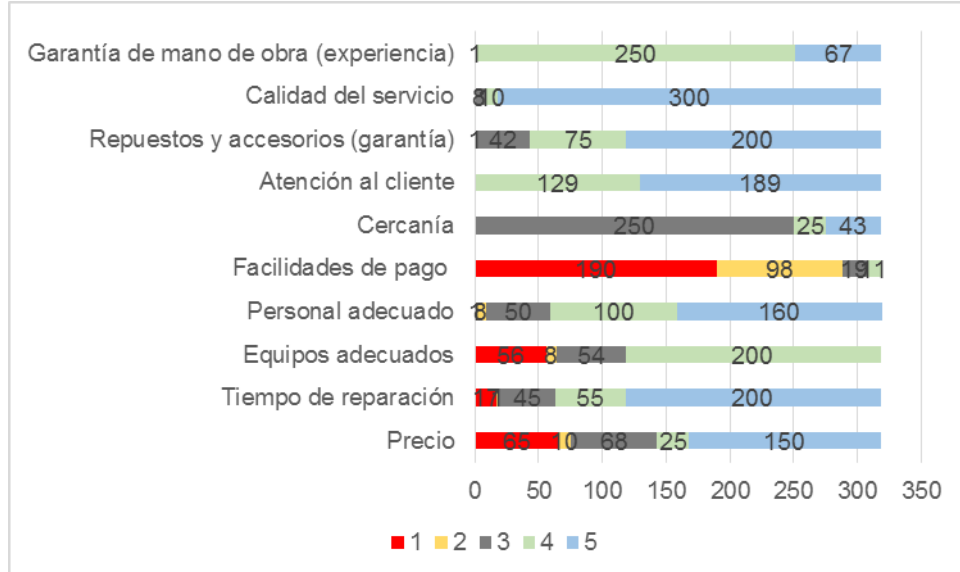

Gráfico 2. Aspectos que consideran importantes Fuente: Autor 
Un dato adicional que se ha manifestado en la búsqueda de información es que los medios de comunicación por donde la población se mantiene informada es la Televisión, seguido por la radio, prensa, internet, vallas publicitarias y propagandas.

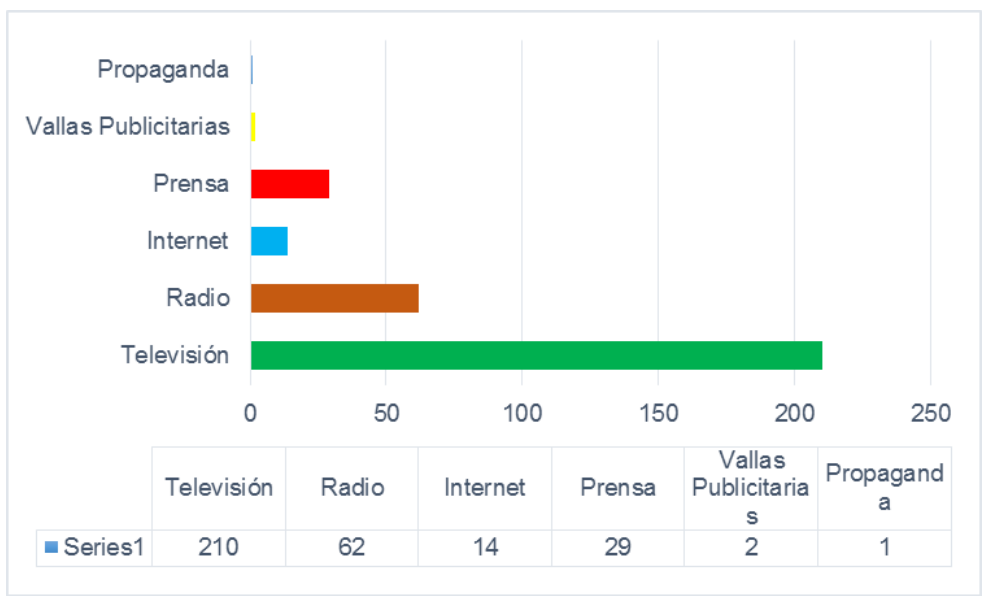

Gráfico 3. Medios de comunicación Fuente: Autores

\section{Conclusión}

Las empresas, emprendedores (maestros, artesano, estudiantes) que tengan conocimientos acerca de estas actividades pueden incursionar a Barbones como una de las parroquia que puede brindar estos servicios pues está en la capacidad de que se pueda ofrecer toda asistencia a lo relacionado con lo automotriz y de línea Blanca, ya que es muy concurrida y visitada por diferentes vehículos ya sean estos pesados y livianos ya que está rodeada de zonas turísticas y empresas bananeras establecidas a su alrededor, además la población cuenta con al menos un modelo de electrodomésticos para brindar este servicio.

Para incrementar estos servicios en la parroquia es de gran importancia conocer a profundidad a la población y aspectos relevantes que estos puedan brindar para que la empresa se mantenga en el tiempo, es por ello que la investigación realizada y plasmada en este documentos nos da a conocer que la necesidad que existe en cuanto a servicios automotrices y de línea blanca es de gran cogida por toda población.

Entre los datos obtenidos en la entrevista, encuesta y observación se conoce que la población se fija en la calidad del servicio que estos ofrecen para visitarlos, también por los repuestos, tiempo de espera, atención al cliente, por el personal Adecuado, garantía, y cercanía entre otros aspectos, además se considera importante la forma de publicitar a la empresa y a sus servicios, ya que la población investigada utiliza de forma periódica los medios comunicación como lo es la Televisión, seguido por la radio, prensa e internet, este dato puede variar para la empresa dependiendo de qué gastos considere implementar para la publicidad.

A pesar de que en la parroquia Barbones están establecidos cinco talleres de iguales servicios, estos no tienen un espacio físico adecuado para dar una buena atención a los clientes debido a los espacios muy reducidos, algunos utilizan parte de la vía pública para brindar estos 
servicios provocando malestar a los transeúntes, es necesario que estos talleres utilicen un espacio adecuado y amplio para brindar mayor comodidad a quienes hagan uso de la empresa.

Como el tema de investigación radica en realizar un diagnóstico para verificar si es factible implementar servicios automotores y de línea blanca, se realizaron diversas formas para recolectar información como encuesta, entrevista y guía de observación, con los resultados se pudo observar que la demanda por arreglo vehicular en ABC motor inyección cada vez incrementa más, de la misma manera existe una fuerte demanda en arreglo de cocinas eléctricas y a gas, con estas respuesta se pudo establecer que la hipótesis número uno en efecto es positiva, de la misma manera la siguiente hipótesis también es positiva ya que se evidenció que aunque la demanda incremente la mano de obra no es la calificada para ejecutar estas actividades, es por esto que se recomienda capacitar al personal para que este apto a ejecutar las actividades encomendadas y adecuar espacios físicos que permitan brindar estos servicios sin incomodar a la ciudadanía.

\section{Bibliografía}

Acs, Z., \& k, K. (2007). State of Literature on Small and Medium - Size Enterprises and Entrepreneurship in Low - Income Communities. Max Planck Institute of Economics, Discussion Papers on Entrepreneurship. Growth and Public Policy N. 307,

Aldana de Vega, L. (2011). Calidad de Servicios (2a. ed ed.). Bogotá: Ecoe. Ediciones. Recuperado el Agosto de 2017, de http://basesdedatos.utmachala.edu.ec:2051/lib/utmachalasp/reader.action?docID=10552805\#

Antiche, J. (2012). El Laboratorio - taller de mecánica automotriz como espacio de aprendizaje en las escuelas técnicas. (Vol. Volumen $16 \mathrm{~N}^{\circ} 1$ ). Venezuela.

Avecevo Ibañez, A., \& López M., A. F. (2000). El Proceso de la Entrevista. México: Limusa. Obtenido de https://books.google.es/books?hl=es\&lr=\&id=VWi4_aHmKAC\&oi=fnd\&pg=PA7\&dq=concepto+entrevista\&ots=iwaMdISJ7m\&sig=tHh5 WMvZOUwrkUEb5tBZ4EMoC6k\#v=onepage $\& \mathrm{q}=$ concepto\%20entrevista $\& \mathrm{f}=$ false

Bernardo Hernando, L. (2008). Seguridad en el mantenimiento de vehículos, segunda edición. (C. M. Rojo, Ed.) Madrid: Ediciones Paraninto S.A.

Borda Pérez, M., Tuesca Molina, R., \& Navarro Lechuga, E. (2013). Métodos Cuantitativos. Bogotá: Editorial Universidad del Norte. Obtenido de http://basesdedatos.utmachala.edu.ec:2051/lib/utmachalasp/reader.action?docID=10844775

Chacón A., L. (2004). Técnología Mecánica 1. Máquinas y Herramientas. México: Editoriar Limusa S.A de C.V.

Ecuador, P. (2013). Análisis del sector automotriz. Guayaquil: Direccion de Inteligencia Comercial e Inversiones. .

Ecuador, P. (2017). Análisis Sectorial Automotriz. Quito: Instituto de Promoción de Exportaciones e Inversiones. 
Española, R. A. (s.n). DLE. Obtenido de DLE: http://dle.rae.es/?id=XhXvJqs

GADPT. (Mayo de 2015). Plan de desarrollo y ordenamiento territorial de la parroquia Tendales. Obtenido de Plan de desarrollo y ordenamiento territorial de la parroquia Tendales.: http://app.sni.gob.ec/snilink/sni/PORTAL_SNI/data_sigad_plus/sigadplusdiagnostico/0760028270001_Diagn\%C3\% B3stico_28-10-2015_17-24-08.pdf

Gallardo Vázquez, P., \& Camacho Herrera, J. M. (2008). La investigación Participativa y su aplicación en el campo Social y Educativo. Wanceulen. Obtenido de http://basesdedatos.utmachala.edu.ec:2051/lib/utmachalasp/reader.action?docID=11231467

HernandezSampieri, R. (2014). Metodología (Vol. VI). Obtenido de http://upla.edu.pe/portal/wpcontent/uploads/2017/01/Hern\%C3\%A1ndez-R.-2014-Metodologia-de-laInvestigacion.pdf.pdf

Krajewski, L. J., \& Ritzman, L. P. (2000). Adminstración de Operaciones, Estrátegia y análisis. México. Obtenido de https://books.google.com.ec/books?id=B6LAqCoPSeoC\&printsec=frontcover \&hl=es\#v=one page $\& q \& \mathrm{f}=$ false

López, R., López, B., \& Bernabeu, N. (2012). La entrevista y la crónica. Mediascopio. Obtenido de http://site.ebrary.com/lib/utmachalasp/reader.action?docID=10664874

Pastor, A., Escobar, D., Mayoral, E., \& Ruiz, F. (2010). Cultura General. Madrid: Ediciones Parainfo S.A. Obtenido de https://books.google.com.ec/books?id=YG6ktKlAm34C\&printsec=frontcover\&hl=es\#v=one page $\& q \& \mathrm{f}=$ false

Quiñones, M. E., \& Aldana de Vega, L. (2006). Calidad y Servicios, Conceptos y Herraminetas. Bogotá.

Quisiguiña, C. (2016). Evolución del sector automotriz 2011 - 2015. Quito: Banco Central del Ecuador.

Saavedra G, M., \& Hernandéz C, Y. (2008). Caracterización e importancia de las MIPYMES en Latinoamérica: Un estudio comparativo. Actualidad Contable FACES, pág: 132,133.

sni.gob. (Mayo de 2015). Sistema Nacional de Información. Obtenido de Sistema Nacional de Información:

http://app.sni.gob.ec/snilink/sni/PORTAL_SNI/data_sigad_plus/sigadplusdiagnostico/0760028270001_Diagn\%C3\% B3stico_28-10-2015_17-24-08.pdf 